\section{REVISTA}

Actualidades Investigativas en Educación http://revista.inie.ucr.ac.cr/

ISSN 1409-4703

UNIVERSIDAD ABIERTA A LAS PERSONAS ADULTAS MAYORES: ESPACIO DE PEDAGOGÍA SOCIAL, EDUCACIÓN Y EMPODERAMIENTO

OPEN UNIVERSITY FOR THE THIRD AGE: SPACE OF SOCIAL PEDAGOGY, EDUCATION AND EMPOWERMENT

Volumen 15, Número 3

Setiembre - Diciembre

pp. $1-18$

Este número se publicó el $1^{\circ}$ de setiembre de 2015

DOI: http://dx.doi.org/10.15517/aie.v15i3.20672

Rita de Cássia da Silva Oliveira

Paola Andressa Scortegagna

Revista indizada en REDALYC, $\underline{\text { SCIELO }}$

Revista distribuida en las bases de datos:

CATÁLOGO DE LATINDEX, IRESIE, CLASE, DIALNET, DOAJ, E-REVIST@S, SHERPA/ROMEO, QUALIS, MIAR

Revista registrada en los directorios:

ULRICH'S, REDIE, RINACE, OEI, MAESTROTECA, PREAL, CLACSO

Los contenidos de este artículo están bajo una licencia Creative Commons 


\title{
UNIVERSIDAD ABIERTA A LAS PERSONAS ADULTAS MAYORES: ESPACIO DE PEDAGOGÍA SOCIAL, EDUCACIÓN Y EMPODERAMIENTO
}

\author{
OPEN UNIVERSITY FOR THE THIRD AGE: SPACE OF SOCIAL PEDAGOGY, EDUCATION \\ AND EMPOWERMENT
}

\section{Rita de Cássia da Silva Oliveira ${ }^{1}$ Paola Andressa Scortegagna ${ }^{2}$}

\begin{abstract}
Resumen: Este ensayo presenta una reflexión fundamentada en una investigación documental sobre la inserción de la persona adulta mayor en espacios educacionales (universidades abiertas), que privilegien la Pedagogía Social, como medio de educación no formal y educación permanente, para posibilitar el empoderamiento. El resultado muestra que la educación para la persona mayor debe posibilitar inserción social, reconocimiento de papeles sociales, actualización de conocimientos, valorización y elevación de la autoestima, calidad de vida, dignidad y ciudadanía. En las últimas décadas se acentúa el envejecimiento de la población, el cual es considerado como uno de los grandes desafíos de la actualidad. Así surge la necesidad de políticas públicas y acciones que se dirijan a la atención de las demandas de este grupo etario para procurar la mejora de las condiciones de vida, garantía de los derechos y el empoderamiento mediante una educación pautada en la Pedagogía Social.
\end{abstract}

Palabras clave: PEDAGOGÍA SOCIAL, EDUCACIÓN NO-FORMAL, POBLACIÓN ADULTA MAYOR, POLÍTICA PÚBLICA, BRASIL.

Abstract: This paper presents a reflection based on documentary research on the integration of Older Adults in educational spaces (open universities), Social Pedagogy that privilege as a means of nonformal education and lifelong learning, to enable empowerment. The result shows that senior education should enable social inclusion, recognition of social roles, update knowledge, recovery and raising selfesteem, quality of life, dignity and citizenship. In recent decades, is accentuated population aging, which is considered one of the greatest challenges today is emphasized. Thus the need for public policies and actions aimed to address the demands of this age group to ensure improved living conditions, safeguarding the rights and empowerment through education in Social Pedagogy scheduled arises.

Keywords: SOCIAL PEDAGOGY, NON-FORMAL EDUCATION, ELDERLY POPULATION, PUBLIC POLICIES, BRAZIL

\footnotetext{
1 Profesora del Departamento de Educación y del Programa de Postgrado en Educación de la Universidade Estadual de Ponta Grossa (UEPG), Brasil (Maestría y Doctorado). Coordinadora del Programa Universidad Abierta para la Tercera Edad (UEPG). Dirección electrónica: soliveira13@uol.com.br

2 Profesora del Departamento de Pedagogía de la Universidade Estadual de Ponta Grossa, Brasil. Profesora del Programa Universidad Abierta para la Tercera Edad (UEPG). Dirección electrónica: paola scortegagna@hotmail.com
}

Ensayo recibido: 29 de noviembre, 2014

Enviado a corrección: 7 de mayo, 2015

Aprobado: 13 de julio, 2015 


\section{Introducción}

Con el cambio de panorama demográfico mundial debido al crecimiento significativo de adultos mayores, este segmento etario ha sido objeto de investigación en diferentes áreas de conocimiento. Como consecuencia surge una demanda por nuevas acciones y políticas que vengan a atender las necesidades de esta población. Actualmente, la longevidad se configura como uno de los grandes desafíos, asumiendo cada vez más un papel relevante en la sociedad brasileña.

A pesar de todos los prejuicios que revisten a la vejez y al adulto mayor, hay una comprensión distinta en el campo de investigación de la gerontología que considera este sujeto como activo, que tiene condiciones de aprender al largo de su vida y encontrar nuevos papeles sociales en su casa o en su comunidad.

La persona mayor tiene innumerables derechos, pero muchas veces existe desconocimiento sobre cuáles son, principalmente, los relacionados con la cuestión educacional. A pesar de ello, se sabe que en diversas situaciones no existen las mínimas condiciones de supervivencia, los derechos básicos no son respetados y la mayoría se encuentra a la deriva en la sociedad.

Debido a un panorama hostil que la sociedad capitalista muchas veces impone a la persona mayor, aparece la necesidad de políticas públicas que atiendan sus demandas, asegurando que los derechos básicos sean garantizados. Así, las políticas públicas volcadas hacia la persona mayor procuran reconocer derechos para un público que se encuentra vulnerabilizado.

Además de políticas públicas generales para la persona mayor, existe un recorte que hace referencia al derecho a la educación, y este como cualquier otro derecho debe ser respetado. En el ordenamiento jurídico brasileño no existe una política educacional para la persona mayor, sino políticas públicas generales, que presentan artículos con prescripciones sobre la educación para esta población.

En este sentido, las Universidades Abiertas a las Personas Adultas Mayores surgen como posibilidad de inserción de la persona mayor en un espacio educacional no formal, que busca la integración social, adquisición de conocimientos, elevación de la autoestima, valorización personal, conocimiento de los derechos y deberes y ejercicio pleno de la ciudadanía. Se destaca también la no formalidad del currículo, que se organiza de manera más interactiva, caracterizando la pedagogía social. 
El trabajo tuvo por objetivos reflexionar sobre la educación como posibilidad de empoderamiento de las personas adultas mayores, identificar las Universidades Abiertas para las Personas Adultas Mayores como espacio de educación no formal y de intervención de la pedagogía social volcada al segmento de la tercera edad.

En relación con los procedimientos metodológicos, se estructura esta investigación cualitativa, a través de una revisión bibliográfica, considerando los aportes de autores como Beauvoir (1990), Both (2003), Furter (1976), Moody (2008), Oakley y Clayton (2003) y Oliveira (1999). La delimitación a estos autores hace referencia al contexto brasileño, en especial, a la convergencia de las investigaciones en el área. Estos autores fueron elegidos porque responden a los objetivos de la investigación.

\section{Pedagogía Social y empoderamiento}

La valorización y respeto al adulto mayor surge como necesidad apremiante en la distribución más equitativa del poder, posibilitando una perspectiva de ruptura con la discriminación generalizada atribuida a este segmento. El poder y su ejercicio cotidiano asumen como concepto central en los procesos de transformación, desarrollo y promoción de la ciudadanía de los individuos.

El poder puede ser entendido como un proceso de concientización, en el cual el individuo interactúa con su contexto social, se conoce a si mismo y al mundo, transformándose al mismo tiempo en que es transformado por el mundo. Esta mayor criticidad y visión del mundo posibilita una mayor capacidad de intervención y control de las situaciones. El mayor se encuentra entre los grupos que son marginalizados y oprimidos caracterizados por lo que Freire (2005) llama "cultura del silencio". En esa dirección se produce la transformación social entre los que detentan o no el poder y resulta en una distribución con mayor equidad.

En esta nueva visión, los adultos mayores van a desempeñar un papel más activo, desarrollando las habilidades y asumiendo la postura de protagonistas legítimos del propio desarrollo (Oakley y Clayton, 2003).

Según Oliveira, Scortegagna y Oliveira (2010, p. 5),

Se pueden distinguir cuatro formas básicas de poder: cultural, social, político y económico. Estos diferentes trajes vestidos por el poder no se excluye, al contrario, se complementan y benefician en su conjunto el progreso del individuo, de la familia o del grupo. Compete a cada uno de los grupos sociales promover su propio desarrollo, 
entendiendo que desarrollo es la distribución más equilibrada del poder entre los individuos en la sociedad.

Para que un grupo se empodere, no es necesario que otros grupos disminuyan o pierdan su poder. Si tenemos en cuenta que hoy en día vivimos en la era de empoderamiento. Implica que otros grupos que están al margen del poder necesitan apoyo para empoderarse a sí mismos, para tener posibilidades de adquisición de conocimientos y para ser socialmente activos y respetados.

“Tanto 'empoderados' como 'desempoderados' son categorías de actores fundamentales para la comprensión de la dinámica en cualquier proceso de desarrollo", en este sentido "el poder define el patrón básico de las relaciones económicas y sociales en un contexto dado, y por lo tanto, tiene influencia fundamental en cualquier intervención que potencialmente amenace a la distribución existente" (Oakley y Clayton, 2003, p. 10).

Los mayores en la sociedad brasileña son considerados desempoderados porque son victimizados culturalmente, resultado de la vulnerabilidad reforzada por prejuicios y estereotipos negativos relativos a la vejez. Mientras tanto ellos se organizan y movilizan para adquirir mayor reconocimiento social, y así pasan a constituir un desafío a las estructuras existentes.

"El poder también está relacionado con el conocimiento, el cual consiste en una fuente de poder y una forma de adquirirlo" (Oakley y Clayton, 2003, p. 11). Así, todo conocimiento adquirido puede interferir y alterar las relaciones existentes, en la medida en que este legitima la autoridad. Contraponiéndose a esto, la ausencia de conocimiento acarrea carencia de poder. No significa que para que el grupo de adultos mayores adquiera poder, necesariamente precisa ocurrir una reducción de poder por parte de otros grupos, mas seguramente el empoderamiento de este grupo resultará en consecuencias sobre el poder ejercido por los demás grupos.

El conocimiento pasa a ser un instrumento eficiente y necesario para el empoderamiento, en especial para los mayores, en la tentativa de superar los desequilibrios sociales.

No existe en la sociedad brasileña una cultura del envejecimiento. De esta manera, se torna malo ser una persona mayor, ya que durante esta etapa de la vida se vuelven vulnerables y se enfrentan a prejuicios y estereotipos negativos (no comprobados científicamente). 
La vulnerabilidad social de las personas mayores suele asociarse, en las sociedades capitalistas, a sujetos socialmente improductivos, con difícil acceso al mercado laboral, y quienes lo logran lo hacen de manera informal o sometidos a trabajos de segunda calidad y bajos salarios. Esta situación se ve reforzada por la incapacidad de muchas personas mayores de ser económicamente independientes o no alcanzar una autonomía social e incluso simbólica.

Según Carvalho y Gastaldo (2008) hay dos abordajes del empoderamiento, a saber: el enfoque psicológico y el enfoque comunitario o social. El enfoque psicológico aborda más el aspecto individual de la transformación y la autonomía. Este enfoque considera a importancia de la autoestima, como también la mayor participación en los grupos de convivencia de la persona mayor, como la familia, amigos, iglesia y clubes sociales. En este enfoque hay destaque para la participación individual a través de un mayor control sobre la propia vida.

Así, el empoderamiento transforma a cada persona en protagonista de su historia, que interviene, participa, influye y transforma el entorno en el que vive.

De acuerdo con Lappe y Du Bois (1984), el empoderamiento individual se manifiesta en la participación política y en la conciencia un sentido de la militancia no se basa en un pequeño interés personal, ya sea en la sensación de una víctima, o echar la culpa a los demás, sino en el sentido más amplio de la comunidad y la responsabilidad". Hay que considerar que el empoderamiento psicológico (individual) ejerce gran influencia sobre el empoderamiento colectivo, comunitario o social.

El empoderamiento comunitario y social se refiere al modo en que las comunidades alcanzan la equidad de recursos, identificando problemas y soluciones, presentando igualdad y capacidad para la resolución de problemas individuales y colectivos. Implica procesos que promueven la participación con el propósito de aumentar el control sobre la vida por parte de los individuos y comunidades, la eficacia política, una mayor justicia social y la mejoría de la calidad de vida (Pinheiro y Miranda, 2010).

El empoderamiento individual ayuda a las personas a conquistar su propia autonomía, el control de su vida, el ejercicio de la ciudadanía a través del desarrollo de sus capacidades, habilidades y competencias individuales, pero que influye en lo colectivo, propiciando una mayor articulación con sus pares, en diferentes situaciones, articulando y movilizando a la comunidad en la búsqueda del empoderamiento colectivo. 
Paulo Freire defiende la idea de empoderamiento de los individuos a través del proceso de concientización política y ciudadana, de la educación dialógica, del conocimiento del mundo en que cada uno se inserta y a través de la crítica y del reconocimiento de este contexto, cada uno pueda insertarse y actuar para el desarrollo de si y del mundo. La educación debe ser vista como práctica liberadora, superando la visión tradicional de educación, en la cual el alumno es solamente receptor de informaciones y conocimientos, sin intervenir, fortaleciendo una relación vertical en que el profesor ejerce el poder sobre los alumnos, la educación bancaria citada por Freire (1996, 2005). Cabe recordar que según el referido autor, nadie enseña a nadie, porque el proceso de aprender es recíproco, así yo mientras enseño, también aprendo y viceversa.

El conocimiento pasa a ser un instrumento eficiente y necesario para el empoderamiento, en especial para los mayores, en la tentativa de superar los desequilibrios sociales.

\section{Educación: un derecho del adulto mayor}

Uno de los grandes desafíos para este siglo es el envejecimiento de la población y cómo las diferentes sociedades deben prepararse para asumir esta realidad que ya es evidente.

Se va esbozando, lentamente, un nuevo paradigma para la vejez. Pero, todavía deben hacerse efectivas políticas públicas y acciones prácticas para que este cambio cultural se produzca, con otra concepción de vejez, en que la persona mayor es activa, participativa, integrada y ejerciendo su ciudadanía, que supera discriminaciones, prejuicios que hoy son rutinarios y no pocas veces enaltecidos.

Simone de Beauvoir ya retrataba en su libro ese panorama hostil en relación con la persona mayor, cuando afirma:

He aquí por qué escribo este libro: para quebrar la conspiración del silencio [...]. Es preciso perturbar su tranquilidad. Con relación a las personas mayores, esta sociedad no es solamente culpable, sino criminal. Amparada en los mitos de la expansión y de la abundancia, trata a los viejos como parias. (Beauvoir, 1990, p. 8)

Las personas adultas mayores tienen hoy visibilidad en la sociedad, en los medios, en la búsqueda de políticas públicas, se volvieron objeto de estudios e investigaciones 
científicas, en una tentativa de superar el enaltecimiento de la juventud en detrimento de la vejez.

Este cambio de mentalidad sobre la vejez como fenómeno natural, asociada a otros factores que propician una ampliación no sólo de años de vida, sino también de calidad de vida debe combatir situaciones discriminatorias y prejuiciosas, que considera la singularidad y las potencialidades de cada individuo a lo largo de su vida.

Al tener en cuenta que el hombre es un ser inconcluso, que continuamente está desarrollándose, creciendo, perfeccionándose, puede considerarse a la educación como un proceso indispensable a lo largo de la vida, considerando aquí principalmente la cuestión de la educación y del proceso de enseñanza y aprendizaje en la fase de la vejez.

Los mayores cada vez más, frente a esta transformación demográfica, se concientizan de su propia posición y del papel que ocupan en la sociedad, rehusándose a permanecer restringidos a ambientes sin gran participación social, o sin estímulos para desarrollarse, o ser recordados con limitaciones o pérdidas, al contrario, reclaman y reivindican mayor reconocimiento social, actúan en diferentes espacios públicos y virtuales, con el propósito de conseguir mayor visibilidad y derivada de ella, más atención y reflexiones sobre la vejez, más políticas públicas con acciones prácticas, superación de prejuicios, mayor valoración y respeto.

Muchas instituciones están preocupadas con el envejecimiento poblacional y ofrecen alternativas en el área de la educación para acoger a esta franja etaria y brindarles herramientas con conocimientos, informaciones que los actualicen y favorezcan una mayor participación y ejercicio de la ciudadanía. Las universidades, cada vez más, abren un espacio para el adulto mayor, superando barreras y socializando los conocimientos producidos.

La educación pregonada por la UNESCO, como educación a lo largo de la vida, le posibilita a la persona mayor más valoración, proporciona condiciones para que desarrolle su autonomía, favorece el ejercicio de nuevos papeles sociales por parte de este segmento etario, posibilitando una mayor inserción y participación social.

Según el Estatuto do Idoso ${ }^{3}$ (Ley 10741/03), en el Capítulo 5, en los artículos del 20 al 25, se establece que el adulto mayor tiene derecho a la educación, respetando la peculiar condición de su edad. El Poder Público creará oportunidades de acceso de la persona

\footnotetext{
${ }^{3}$ En Brasil la utilización del término "idoso" es común y hace referencia al grupo etario de las personas adultas mayores o tercera edad. Este artículo presenta su discusión utilizando indistintamente los términos "personas adultas mayores", "adultos mayores" o "mayores".
} 
mayor a la educación, abriendo cursos especiales para que el mismo se integre a la vida moderna, además de apoyar la creación de universidades abiertas a las personas mayores y publicaciones de libros y periódicos con contenidos adecuados a la población de edad avanzada.

Cumpliendo con la legislación brasileña, en especial con el Estatuto do Idoso, y las funciones que son atribuidas a las universidades: enseñanza, investigación y extensión, son ofrecidos por las Instituciones de Enseñanza Superior programas como formas alternativas de atención al adulto mayor y también a los individuos que van a envejecer, procurando además de una valorización, una mayor concientización de la sociedad en general sobre el proceso de envejecimiento de la población en el país.

Los programas para la tercera edad no deben asumir una connotación meramente asistencialista o de ocio porque de cierta manera no dejan de ser una forma sutil de marginalizar y alienar a los mayores de la sociedad. Debe ser privilegiado el aprendizaje, por un lado confrontándoselo con el quiebre del prejuicio de que el mayor tiene menor capacidad de aprendizaje y por otro lado, haciendo surgir el aprendizaje con sabor de conquista, de victoria, elevando la autoestima e imagen del adulto mayor, además de aguzar el sentido de utilidad, mejorando la capacidad crítica, la libertad de expresión y participación de ese segmento de la población.

Muchas universidades abren espacio para los mayores mediante la creación de las Universidades para la Tercera Edad (o Universidades a las Personas Adultas Mayores), haciendo que ellas amplíen su compromiso, buscando integrar a aquellos que se encuentran al margen del proceso de desarrollo (la exclusión ligada a la edad), los lleva a disfrutar de los bienes que llegan con esta propuesta. De esta manera, los adultos mayores se fortalecen a través de grupos articulados, consiguiendo mayor visibilidad y organización en la sociedad.

Los programas volcados a personas de la tercera edad en las universidades, presentan un propósito bien definido: brindarles una mejor calidad de vida. Así, contribuyen a la reformulación del sentido que se da a la vida, posibilitan la ampliación de las relaciones sociales y la producción de conocimientos específicos, que puedan subsidiar políticas pertinentes.

Es indispensable despertar más atención e inversiones por parte del Estado y de la sociedad civil para minimizar la precariedad de vida de los adultos mayores. La persona adulta mayor en Brasil no constituye un hecho económico serio, y así, no atrae mayores inversiones en el sector. Esa situación debe ser revertida por la educación, para ello debe 
ser tratado con respeto y dignidad y precisa no sólo de políticas públicas, sino de acciones, de intervenciones prácticas, educativas, con ética, conciencia y compromiso.

La educación tiene un papel político fundamental, “(...) ella debe desempeñar un papel eminentemente democrático, ser un lugar de encuentro, de permanente intercambio de experiencias" (Gadotti, 1984, p. 157).

La educación, además de un derecho para la persona mayor representa la posibilidad de cambios conceptuales en relación con el envejecimiento y la vejez. Una acción educacional que contemple esta temática estimula la reflexión en relación con el proceso de envejecimiento poblacional, como también facilita la propia aceptación de la condición de la persona mayor.

La educación supera actualmente la idea de reproducción y de transmisión de la herencia acumulada por la humanidad porque asume un carácter de transformación al brindar a los individuos conocimiento técnico y científico, que los permite actuar en las diferentes áreas, porque hay la superación del papel reduccionista de mera transmisión de conocimientos. La educación refleja a la sociedad y ejerce un papel político importante al concientizar a la población, en la medida en que posibilita el desarrollo del espíritu crítico, la creatividad y el intercambio de experiencias.

Según Piconez (2002), la educación instrumentaliza crítica y creativamente, teniendo a la vista la renovación de la realidad. De esta manera, se observa cuánto el proceso educativo facilita un estadio de cambios, independientemente de la edad. La educación es un importante medio de transformación y valorización de estas personas.

El proceso de aprendizaje ocurre durante toda la vida del hombre, siempre en busca de la actualización, adquisición de nuevos conocimientos, en una sociedad globalizada, con cambios continuos y a ritmo acelerado (Oliveira, 1999).

El individuo aprende constantemente en su vida, así, la educación no puede ser caracterizada sólo como una etapa con tiempo y espacio predeterminados. Según Furter (1976) el hombre es un ser inacabado, que busca la perfección, con este sentido la educación se torna un proceso continuo que solo termina con la muerte.

La educación debe ser vista como un proceso, un hecho existencial y social. Es considerada como un fenómeno cultural y no consiste en la formación uniforme de todos los individuos porque se desarrolla sobre el proceso económico de la sociedad. Es en sí una actividad teleológica, siempre persigue un fin, siendo un hecho de orden consciente, un proceso exponencial, con una esencia concreta y de naturaleza contradictoria (Pinto, 1989). 
A toda concepción de educación existe subyacente una visión de mundo, de hombre y de sociedad; basada en estas concepciones se determinan los fines a ser alcanzados por la educación. La educación es una práctica social, situada históricamente, en una realidad concreta, englobando diferentes aspectos culturales, económicos, sociales y políticos.

La educación como práctica social permite que el hombre constantemente aprenda, haciéndose posible pensar en una transformación cultural y en la propia sociedad. Para Pinto (1989, p. 39), "la educación no es una conquista del individuo", sino "una función de la sociedad y como tal dependiente de su grado de desarrollo. Donde hay sociedad hay educación: luego, esta es permanente".

El proceso educativo debe ser reflejado a la luz de los pilares de la educación pregonados por la UNESCO: aprender a ser (la educación debe contribuir al desarrollo total de la persona, espíritu y cuerpo, inteligencia, sensibilidad, sentido estético, responsabilidad personal, espiritualidad; desarrollar la autonomía, o discernimiento y la responsabilidad); aprender a hacer (la educación debe contribuir para que la persona adquiera competencias para una mejor cualificación profesional, experimentando una diversidad de actividades, alternando la enseñanza y el trabajo, teoría y práctica), aprender a conocer (combinando con una cultural general amplia y aprender a aprender para saber aprovechar las oportunidades que la vida personal y profesional le presente); aprender a convivir (desarrollar la comprensión del otro, la percepción y sensibilidad de la interdependencia entre las personas en la sociedad que vivimos) (Delors, et al., 2001).

Entonces, se percibe la necesidad de una acción educacional volcada hacia el adulto mayor, con carácter de educación permanente. Se nota que cuando la educación se vuelve permanente en la vida de la persona, independientemente de la edad, es posible encontrar un nuevo sentido al vivir, aun cuando ya se encuentran desacreditados por la propia sociedad.

La educación a lo largo de toda la vida es una construcción continua de la persona humana, de su saber y de sus aptitudes, pero también de su capacidad de discernir y actuar. Debe llevarla a tomar consciencia de si misma y del medio que la envuelve y a desempeñar el papel social que le cabe en el mundo del trabajo y en la comunidad. E saber, el saber hacer, el saber vivir juntos y el saber ser constituyen cuatro aspectos íntimamente ligados, de una misma realidad. Experiencia vivida en el cotidiano, y marcada por momentos de tensión, esfuerzo, de comprensión de datos y de hechos complejos la educación a lo largo de toda la vida es el producto de una dialéctica con 
varias dimensiones. $\mathrm{Si}$, por un lado implica la repetición o imitación de gestos y de prácticas, por otro es, también, un proceso de apropiación singular y de creación personal. Junta el conocimiento no formal al conocimiento formal, el desarrollo de aptitudes innatas a la adquisición de nuevas competencias. Implica esfuerzo, pero trae también la alegría de la descubierta. Experiencia singular de cada persona ella es, también, la más compleja de las relaciones sociales, dado que se inscribe al mismo tiempo en el campo cultural, en el laboral y en el de la ciudadanía. (Delors et al., 2001, p. 107)

La educación permanente posibilita el desenvolvimiento del capital humano. De esta manera, la misma debe estar vinculada para que realmente este capital se desenvuelva independientemente de la clase social o situación de marginalización en que el individuo esté inserido, permitiendo que se produzca el desarrollo intelectual, social, cultural y político.

La educación asume un papel democrático, buscando la transformación social a través de la participación y real integración de los ciudadanos.

Esencial también es luchar por una efectiva democratización de la educación, extendiéndola a todos, de modo de brindar instrumentos a los individuos con los contenidos básicos valorizados por la sociedad, para que, una vez dotados de los instrumentos de acceso al saber y de sentido crítico, puedan tener mejores condiciones de vida y de trabajo. (Oliveira, 2001, p. 20)

De esa manera, Mosquera (1975, p. 141) concluye que "(la) educación permanente y universal en su carácter es esencial para la completa democratización del aprendizaje, caracterizada por su flexibilidad y diversidad de contenidos, aprehendiendo elementos, técnicas y finalidades abiertas al tiempo y al espacio".

La educación ocupa papel fundamental en la formación crítica del mayor, para que tenga condiciones de mantenerse activo y consciente de su propia vejez. Es por medio de la acción pedagógica que se brinda la oportunidad de una mayor inserción social. Además de la formación de la persona mayor como actor social, las articulaciones en red traen la posibilidad de exigir más respeto, dignidad y un compromiso sociopolítico a propósito de sus derechos.

Como afirman Oliveira, Scortegagna e Oliveira (2011, p. 90),

[...] tan fundamental como la ciudadanía es el derecho a la educación, pues no se alcanzará la ciudadanía sin que haya conocimiento pleno de este derecho. Así, pensar 
la educación para la tercera edad, es pensar más que una ocupación para la persona mayor, es permitir una acción intensiva e intencional para que este sujeto se perciba, entienda su entorno social, político y económico, como también para que no sea engañado o tenga sus derechos negados.

La educación es considerada como un derecho fundamental, que está incluido en algunas políticas públicas destinadas al público mayor, aunque todavía no existe ninguna política que referencie exclusivamente la educación a ese grupo. Como práctica social permite que el hombre constantemente aprenda, y sea posible pensar en una transformación cultural y en la propia sociedad.

Relativa a la condición esencial de práctica social, la educación tiene una función esencial con respecto a la persona mayor. los procesos educacionales no pueden estar asociados solamente a la escolarización, sino también a las demás posibilidades, comúnmente encontradas en las prácticas de educación no formal. Y, es en estos espacios que muchas veces el adulto mayor encontrará posibilidad de hablar, efectivizando la participación ciudadana.

\section{El espacio universitario para la educación de los adultos mayores}

Las Universidades Abiertas para las Personas Adultas Mayores (En Brasil: Universidad Abierta para la Tercera Edad - UATI) han sido diseminadas por las diferentes universidades del el mundo como una estrategia de empoderamiento de la persona mayor, una educación no formal y educación permanente al mismo tiempo que le brinda conocimientos, informaciones, posibilitando y legitimando el ejercicio pleno de la ciudadanía.

Las UATIs privilegian la educación no formal caracterizándose por el encuentro de generaciones, la no obligatoriedad de frecuencia, la ocurrencia de acciones y experiencias en espacios y tiempos más flexibles no restringidos a los fijados por órganos reguladores.

La educación no formal, al contrario de la educación formal, se caracteriza por no tener la preocupación de desarrollar un currículo predefinido, un currículo que se hace principalmente basado en deseos, necesidades e intereses de las personas que constituyen los grupos involucrados en acciones y prácticas de ese campo educacional. (Silva, 2006, p. 9) 
Este empoderamiento en diferentes niveles, social, cultural, económico y político a largo plazo posibilitará reequilibrar la estructura de poder dentro de la sociedad y contribuir para elaborar otro paradigma de vejez.

El empoderamiento en Brasil, en los años '90 tuvo como objetivo la búsqueda de la promoción del desarrollo social, abriendo así espacio a la consolidación de diferentes intervenciones prácticas volcadas a las personas mayores, entre ellas las UATI.

Según Gohn (2006) la educación no formal representa varias dimensiones, como el aprendizaje de los derechos desde una perspectiva política, desarrollo de potencialidades, ejercicio de prácticas comunitarias y sociales, aprendizaje que capacite para una lectura de mundo. La educación no formal acontece compartiendo experiencias, en espacios y acciones colectivas y cotidianas."La transmisión de información y formación política y socio cultural es una meta de la educación no formal" (Gohn, 2006, p. 3).

Las UATIs también dan a las personas mayores la posibilidad de ascender en tres grandes áreas de empoderamiento: el poder como mayor confianza en la capacidad personal para llevar adelante algunas formas de acción; el poder como aumento de las relaciones efectivas que las personas desprovistas de poder pueden establecer con otras organizaciones; el poder como resultado de la ampliación del acceso a los recursos económicos, tales como crédito e insumos.

Al pensar a la educación como una acción permanente y al proceso de aprendizaje que ocurre durante toda la vida del hombre, no se evidencia apenas una evolución del pensamiento pedagógico, se trata de una necesidad de constante actualización en un ambiente globalizado donde los cambios son rápidos y continuos, permitiendo que el hombre evolucione según estos preceptos (Oliveira, 1999).

De esta manera, la educación como práctica social permite que el hombre constantemente aprenda, así es posible pensar en una transformación cultural y de la propia sociedad. Para Pinto (1989, p. 39), "la educación no es una conquista del individuo", sino "una función de la sociedad y como tal dependiente de su grado de desarrollo. Donde hay sociedad hay educación: luego, esta es permanente". La educación para la transformación se hace de carácter permanente, tanto para que la sociedad pueda desarrollarse, como para que el individuo pueda estar integrado a este desarrollo.

La sociedad actual está exigiendo una nueva postura del hombre, con más capacidades y conocimientos, y este desarrollo solamente podrá ocurrir a través de una 
acción educacional consciente y que suceda de manera permanente, permitiendo la actualización constante.

Así, "la preocupación básica que orienta el desdoblamiento de educación permanente y desarrollo cultural no se refiere a la continua maduración humana, sino al desarrollo socioeconómico y cultural" (Paiva, 1985, p. 48). Se posibilita entonces que el hombre, en el espacio histórico, cultural, político y social en el que está inserto, consiga evolucionar junto con la sociedad, y esta evolución individual refleja directamente en la constante evolución social.

A través de la educación, el hombre se humaniza, se caracteriza, se torna un ser social y consciente. "La educación es un proceso continuo y permanente que da al ser humano el sentido de la vida, por la posibilidad que establece en la medida en que promueve mayor comprensión sobre el mundo" (Herédia, 2006, p. 126).

La educación permanente representa una necesidad de ampliación de la participación activa del hombre en el contexto social y cultural, con objetivo de mejora en las relaciones interpersonales, en la calidad de vida, en la comprensión de mundo y en la esperanza de futuro mejor y más digno. Con la educación permanente se asume una nueva concepción de vida humana, cuyo principio central no es solo aprender, sino principalmente vivir para aprender, estando integrado e interactuando con quienes se encuentran a su alrededor (Oliveira, 2007).

La educación debe desarrollar al sujeto como un todo, permitiendo que este esté integrado a la sociedad, con una participación activa y consciente. Pensar en un desarrollo integral es lo que conduce a la perspectiva de educación permanente, que persigue la superación de la marginalización social, posibilitando una formación continua, que tenga como principio elemental la práctica social y el hombre en su totalidad.

Para que la educación permanente se produzca de manera satisfactoria se da la necesidad de que esta sea organizada y pensada para que alcance sus fines. Según Furter (1976, p. 127),

La necesidad de una educación continua, que sea una constante en la vida humana y que permita vivir plenamente el mundo planetario, no puede ser resuelta con una simple prolongación de la educación, ni por una mayor carga del campo escolar. Debe tomar a forma de una educación permanente, a partir de la cual deberá ser pensada toda educación, y que obligará a los educadores a inventar nuevas técnicas y nuevos métodos adecuados. 
A partir del momento en que la educación permanente fije sus propósitos y se estructure de manera democrática, un gran salto cualitativo ocurrirá en la educación. El hombre aprende durante toda su vida, sea en espacios escolarizados o no, así, posibilitar que la educación traiga más calidad a los aprendizajes es fundamental.

Para que de hecho se dé una organización de los adultos mayores en busca de la consolidación de sus derechos es fundamental y urgente que la instrumentalización y la educación se hagan posibles para todos. Conocer los propios derechos es precepto elemental para que se reclamen mejores condiciones de supervivencia y una mejor calidad de vida.

Ante estos condicionantes, la educación se presenta como propulsora de la transformación social, pues es a través de ella que, además, de la adquisición de conocimientos, el proceso de socialización se intensifica y la formación de un sujeto crítico y reflexivo se consolida.

A partir del momento en que el mayor es considerado como un sujeto capaz de desarrollar actividades y desempeñar nuevos papeles sociales se percibe que la visión sobre la vejez comienza a ser alterada, pues el adulto mayor incapaz, inútil y sin utilidad, pasa a ser un nuevo agente social. "Gradualmente, la visión de personas mayores como un subgrupo poblacional vulnerable y dependiente fue siendo substituida por la de un segmento poblacional activo y actuante que debe ser incorporado a la búsqueda del bienestar de toda la sociedad" (Camarano y Pasinato, 2004, pp. 257-258).

En esta perspectiva, cuando el adulto mayor se percibe como posibilitador de cambios y que tiene condiciones de actuar a favor de sus derechos, el contexto social de la vejez cambia. Se nota que "los viejos como colectivo social, se vuelven mucho más amenazadores que la vejez como fenómeno biológico" (Fonte, 2002, p. 12).

\section{Conclusiones}

A pesar de todas las conquistas que el segmento de la tercera edad viene alcanzando en los últimos años a través de su representatividad y de las concepciones de envejecimiento activo y constitución de un nuevo actor social, algunos puntos todavía precisan avanzar. Conforme Whitaker (2007, p. 107), "si la persona mayor pierde el poder, todavía tiene derechos. Y por estos se debe continuar luchando. Cuanto menos poder, menos prestigio, mayor debe ser la lucha por los derechos".

Para que de hecho se produzca una organización de las personas mayores en busca de la consolidación de sus derechos es fundamental y urgente que la instrumentalización y la 
educación se hagan accesibles para todos. Conocer los propios derechos es precepto elemental para que se reclamen mejores condiciones de supervivencia y una mejor calidad de vida. La educación ocupa un papel fundamental en la formación crítica de la persona mayor, para que tenga condiciones de mantenerse activo y consciente de su propia vejez. En esta misma perspectiva, es también, a través de la acción pedagógica que se le brinda oportunidad para una mayor inserción social, además de la formación como actor social, que movilizado en red tendrá posibilidad de articulación y pasará a exigir más respeto y dignidad, y un compromiso sociopolítico a propósito de sus derechos.

En este ámbito, se percibe que la educación, además de proponer y ser agente de la acción que transforma, es un derecho elemental de todo ser humano, independiente de la edad, con una percepción educacional permanente. Así, el adulto mayor tiene derecho a la educación, no solo como instrumentalización o compensación, sino como espacio de cuestionamiento, de decisiones, de capacitación y por sobre todo, de diálogo.

Los diferentes programas ofrecidos por las Instituciones de Enseñanza Superior son formas alternativas de atención al mayor, con ello se procura, además de la valorización de esa franja etaria, mayor concientización de la sociedad en general con respecto al proceso de envejecimiento de la población de nuestro país.

Con la inserción de la persona adulta mayor en la comunidad universitaria, la integración entre generaciones se da, necesariamente, fomentando debates sobre las cuestiones que involucren esa franja etaria, analizando prejuicios y discriminaciones sustentadas socialmente y que se presentan sin fundamentación científica.

La persona adulta mayor, al concientizarse de su espacio en la sociedad, tendrá una visión más optimista de si, considerándose productivo, útil, todavía capaz de colaborar mucho con la sociedad en la cual está inserto.

Así, las UATIs se constituyen como espacios de educación no-formal, consideradas como un núcleo fundamental de la Pedagogía Social, la cual se caracteriza por transitar por espacios sociales en los cuales los contornos educativos no son bien delimitados. En estos espacios, el proceso de empoderamiento se hace posible para los adultos mayores, pues por medio de la adquisición de conocimientos y aprendizajes políticos de derechos, este grupo supera una realidad de exclusión y marginalización y se constituye en un grupo social y políticamente activo. 


\section{Referencias}

Beauvoir, Simone. (1990). A velhice. Rio de Janeiro: Nova Fronteira.

Both, Agostinho. (2003). Envelhecimento humano: múltiplos olhares. Passo Fundo: UPF.

Brasil. (2003). Lei no 10741, de 3 de outubro de 2003. Dispõe sobre o Estatuto do Idoso. Brasília.

Camarano, Ana Amélia y Pasinato, Maria Tereza. (2004). Os novos idosos brasileiros: muito além dos 60? Rio de Janeiro: IPEA.

Carvalho, Sérgio Resende, Gastaldo, Denise. (2008). Promoção à saúde e empoderamento: uma reflexão a partir das perspectivas crítico-social pós-estruturalista. Ciência \& Saúde Coletiva, 13, 2009-2040.

Delors, Jacques, Al-Mufti, In'am, Amagi, Isao, Carneiro, Roberto, Chung, Fay, Geremek, Bronislaw... Nanzhao, Zhou. (2001). Educação: um tesouro a descobrir. São Paulo: Cortez/Unesco.

Fonte, Isolina B. (noviembre, 2002). Diretrizes internacionais para o envelhecimento e suas conseqüências no conceito de velhice. Encontro da Associação Brasileira de Estudos Populacionais. In Anais do XII Encontro da Associação Brasileira de Estudos Populacionais, ABEP: Ouro Preto.

Freire, Paulo. (1996). Pedagogia da autonomia. Rio de Janeiro: Paz e Terra.

Freire, Paulo. (2005). Pedagogia do oprimido. Rio de Janeiro: Paz e Terra.

Furter, Pierre. (1976). Educação e vida. Petrópolis: Vozes.

Gadotti, Moacir. (1984). A educación contra a educación. Rio de Janeiro: Paz e Terra.

Gohn, Maria da Glória. (2006). Educação não-formal na pedagogia social. Congresso Internacional de Pedagogia Social, São Paulo, In: Anais do 1ํㅡㄹ Congresso Internacional de Pedagogia Social; Autores sociais. Petrópolis: Vozes.

Herédia, Vânia Beatriz Merlotti. (2006). A família, a educação e o envelhecimento humano: desafios para a sociedade. In: Miriam Bonho Casara, Ivonne Asunta Cortelletti y Agostinho Both (Eds.), Educação e envelhecimento humano (pp. 109-132). Caxias do Sul: EDUCS.

Lape, Frances M. y Dubois, Peter. (1994). Quickeming of America: Rebuilding our Nation Remaking our Lives. San Francisco: Jossey-Bass.

Mosquera, Juan José Mouriño. (1975). Vida adulta: personalidade e desenvolvimento. Porto Alegre: Sulina.

Oakley, Peter y Clayton, Andrew. (2003). Monitoramento e avaliação do empoderamento. Tradução: Zuleika Arashiro e Ricardo Dias Sameshima. São Paulo: Instituto Pólis. 
Oliveira, Rita de Cássia da Silva. (1999). Terceira Idade: do repensar dos limites aos sonhos possíveis. Campinas: Papirus.

Oliveira, Rita de Cássia da Silva. (2007). O processo histórico do estatuto do idoso e a inserção pedagógica na universidade aberta. Revista HISTEDBR Online, Campinas, (28), 278-286.

Oliveira, Rita de Cássia da Silva, Scortegagna, Paola Andressa y Oliveira, Flávia da Silva. (2011). O envelhecimento e a velhice: teorias, demografia e política. Curitiba: CRV.

Oliveira, Rita de Cássia da Silva, Scortegagna, Paola Andressa y Oliveira, Flávia da Silva. (2010). Pedagogia Social: possibilidade de empoderamento para o idoso. Congresso Internacional de Pedagogia Social. In: Anais do 1ํㅡ Congresso Internacional de Pedagogia Social, Associação Brasileira de Educadores Sociais (ABES), São Paulo.

Paiva, Vanilda. (1985). Educação Permanente: ideologia educativa ou necessidade econômico-social? In: Vanilda Paiva y Henrique Rattner (Eds.), Educação permanente e capitalismo tardio (pp. 7-63). São Paulo: Cortez.

Piconez, Stela Conceição Bertholo. (2002). Educação escolar de jovens e adultos. São Paulo: Paulinas.

Pinheiro, Claudia dos Santos y Miranda, Maria Luiza de Jesus. (2010). Empowerment e idosos: uma reflexão sobre programas de educação física. A Terceira Idade, Estudos sobre envelhecimento, 21(48), 7-19.

Pinto, Álvaro Vieira. (1989). Sete lições de educação de adultos. São Paulo: Cortez.

Silva, Roberto. (2006). Fundamentos teóricos e metodológicos da pedagogia social no Brasil. Congresso Internacional de Pedagogia Social, 1, 2006, In: Anais do 1ํ Congresso Internacional de Pedagogia Social, ABEP/USP: São Paulo.

Whitaker, Dulce Consuelo Andreatta. (2007). Envelhecimento e poder. Campinas: Alínea. 